Revista Brasileira de Agricultura Irrigada v.12, nº.5, p. 2834 - 2844, 2018

ISSN 1982-7679 (On-line)

Fortaleza, CE, INOVAGRI - http://www.inovagri.org.br

DOI: $10.7127 /$ rbai.v12n500813

Protocolo 813.18 - 13/11/2017 Aprovado em 22/08/2018

\title{
SALINIDADE E COMPOSIÇÃO CATIÔNICA DO LIXIVIADO DE UM SOLO SALINO-SÓDICO TRATADO COM VINHAÇA
}

\author{
Maria Rosimere Miguel da Silva ${ }^{1}$, Francisco Assis de Oliveira ${ }^{2}$, Lourival Ferreira \\ Cavalcante $^{3}$, Antônio Gustavo de Luna Souto ${ }^{4 *}$, Jefferson Alves Dias ${ }^{5}$, Francisco Thiago \\ Coelho Bezerra ${ }^{6}$
}

\section{RESUMO}

A degradação do solo pelo excesso de sais solúveis em água e de sódio trocável é um dos problemas que mais limitam a produção agrícola das regiões áridas e semiáridas mundiais. Nesse sentido, um experimento foi desenvolvido em ambiente protegido para avaliar o $\mathrm{pH}$, a condutividade elétrica e os teores de cátions do lixiviado de um solo salino-sódico tratado com doses de vinhaça em períodos de incubação. Os tratamentos foram distribuídos em blocos casualizados, no esquema fatorial $5 \times 3$, referente a cinco doses de vinhaça nas concentrações de 20,40, 60, 80, 100 \% e períodos de incubação da vinhaça no solo de 10, 30 e 50 dias. O insumo orgânico promoveu a lixiviação de sais constatado pelo aumento da condutividade elétrica e teores de cátions, principalmente de sódio, e redução do $\mathrm{pH}$ do lixiviado com o aumento do período de incubação da vinhaça ao solo salino - sódico. $\mathrm{O}$ periodo de incubação da vinhaça de dez dias foi o que promoveu maior redução de pH e de condutividade elétrica do lixiviado na dose de $100 \%$ do insumo orgânico. No mesmo período de incubação, as maiores reduções de potássio, sódio, cálcio e magnésio foram 58, 95 e 100\%, respectivamente para cálcio e magnésio.

Palavras-chave: sodicidade, sais, insumo orgânico, composição do lixiviado.

\footnotetext{
${ }^{1}$ Mestre em Manejo de Solo e Água, Universidade Federal da Paraíba, Areia, Paraíba, Brasil. E-mail: rosy.ms@hotmail.com

2 Professor do Instituto Avançado de Ensino Superior de Barreiras, Barreiras, Bahia, Brasil. E-mail: oliveirachico@yahoo.com.br

${ }^{3}$ Professor do Programa de Pós-Graduação em Agronomia e Pesquisador do Instituto Nacional de Ciência e Tecnologia em Salinidade, Universidade Federal da Paraíba, Areia, Paraíba, Brasil. E-mail: lofeca1946@yahoo.com.br

${ }^{4}$ Pós-Doutorando Júnior/CNPq, Universidade Federal da Paraíba, Areia, Paraíba, Brasil. E-mail: gusluso@hotmail.com

${ }^{5}$ Doutorando em Agronomia, Universidade Federal da Paraíba, Areia, Paraíba, Brasil. E-mail: jefferson_cz@hotmail.com

${ }^{6}$ Pós-Doutorando/CAPES, Universidade Federal da Paraíba, Areia, Paraíba, Brasil. E-mail: bezerra_ftc@yahoo.com.br
} 


\title{
SALINITY AND CATIONIC COMPOSITION OF LEACHED SALINE-SODIC SOIL TREATED WITH VINASSE
}

\begin{abstract}
The degradation of the soil by excess salts soluble in water and exchangeable sodium is the problem that most limits the agricultural production of the arid and semi-arid regions of the world, including the Northeast of Brazil. In this sense, an experiment was carried out in a protected environment to evaluate the $\mathrm{pH}$, electrical conductivity and cation contents of the leachate from a saline-sodic soil treated with vinasse doses at incubation periods. The treatments were distributed in randomized blocks, in a $5 \times 3$ factorial scheme, referring to five doses of vinasse at concentrations of $20,40,60,80,100 \%$ and vinasse incubation periods in the soil of 10, 30 and 50 days. The organic matter promoted the leaching of salts as evidenced by the increase in the electrical conductivity and cation contents, mainly of sodium, and the reduction of the $\mathrm{pH}$ of the leachate with the increase of the period of incubation of the vinasse to the saline - sodic soil. The incubation period of the ten day vinasse was the one that promoted greater reduction of $\mathrm{pH}$ and electrical conductivity of the leachate in the dose of $100 \%$ of the organic input. In the same incubation period the highest reductions of potassium, sodium, calcium and magnesium were 58, 95 and 100\% respectively for calcium and magnesium.
\end{abstract}

Keywords: sodicity, salts, organic input, chemical composition.

\section{INTRODUÇÃO}

A salinidade e sodicidade constituem os maiores problemas aos solos agrícolas e resultam na perda da capacidade produtiva das terras nos perímetros irrigados, sobretudo em regiões áridas e semiáridas do mundo (YAZDANPANAHET al., 2011; ABDELFATTAH, 2012). Segundo a Food Agriculture Organization - FAO para o Serviço de Manejo e Nutrição de Plantas, aproximadamente $6 \%$ do total das áreas agricultáveis do mundo estão comprometidas pela salinidade e/ou sodicidade e desse total 32 milhões de hectares sob sequeiro e 45 milhões de hectares sob irrigação exibem algum problema dessa natureza salina (PARIHAR et al., 2015).

No Nordeste brasileiro, a situação dos problemas de sais não é diferente do restante do mundo, devido grande parte do território ser de clima semiárido e apresentar nível de salinidade e sodicidade edáfica ou hídrica que compromete o sistema de produção (RIBEIRO et al., 2016). Essas inconveniências resultam em perdas da produção agrícola culminando, na maioria das vezes, no abandono de terras, antes produtivas, com sérios impactos negativos econômicos e sociais (SOUSA et al., 2014). Os acúmulos progressivos de sais solúveis e/ou sódio trocável promovem a dispersão das argilas com reflexos negativos na capacidade produtiva do solo pela elevação do $\mathrm{pH}$, da condutividade elétrica e o aumento de sódio trocável no complexo sortivo e complexo argila-húmus provocando perdas de permeabilidade, do espaço poroso e aumento retenção de água pelo solo, comprometendo o crescimento e rendimento das culturas (PRAPAGAR et al., 2012).

A recuperação de solos degradados pela salinidade, solos salino-sódicos e sódicos está associada à aplicação de corretivos químicos minerais e também adição de matéria orgânica de origem animal e vegetal seguida de lixiviação e drenagem (GOMES et al., 2000; MIRANDA et al., 2011; PRAPARGAR et al., 2012; SOUSA et al., 2014). Esses insumos, como inclusive a vinhaça, disponibilizam, além de outros nutrientes, o cálcio que substitui o sódio adsorvido ao complexo sortivo e desloca para a solução do solo que em seguida é removido da zona radicular pela ação da lixiviação e drenagem (PESSOA et al., 2010; YAZDANPANAH et al., 2011; 
VASCONCELOS et al., 2016). Entretanto, independentemente do corretivo ser de natureza mineral ou orgânica, a dosagem de cada tipo dever ser fornecida de modo a não provocar outros riscos ambientais, além da salinidade (MIRANDA et al., 2011; PRAPARGAR et al., 2012).

Dentre os corretivos químicos minerais utilizados para redução da sodicidade do solo, expressa pela percentagem de sódio trocável (PST), os mais frequentes são gesso agrícola e cloreto de cálcio, respectivamente, pela maior disponibilidade no mercado e maior eficiência (CAVALCANTE et al., 2016) e dentre os insumos orgânicos de origem animal, o esterco bovino e de origem vegetal a vinhaça que é o principal subproduto da industrialização da cana-deaçúcar - Saccharum officinarum (JALALI; RANJBAR, 2009; YAZDANPANAH; MAHMOODABADI, 2013; NEGUIM; MUSTAFA, 2016).

A vinhaça é mais frequentemente aplicada via fertirrigação na lavoura da canade-açúcar em solos não salinos (BRITO et al., 2007; NICOCHELLI et al., 2012) e em menor proporção na redução da salinidade, sodicidade e alcalinidade dos solos degradados por sais (GOMES et al., 2000; SANTOS et al., 2005; BUENO et al., 2009). Para os respectivos autores, em ambas as situações, a vinhaça é utilizada devida ser fonte de cálcio, magnésio, potássio e matéria orgânica para elevar a melhoria da fertilidade dos solos não salinos e também por reduzir a salinidade, sodicidade e alcalinidade e exercer melhoria física dos solos comprometidos pelos sais.
Pelo exposto, o trabalho teve como objetivo avaliar os efeitos de doses de vinhaça em períodos distintos de incorporação no $\mathrm{pH}$, condutividade elétrica e composição catiônica do lixiviado de um solo salino-sódico.

\section{MATERIAL E MÉTODOS}

O experimento foi conduzido em ambiente protegido do Departamento de Solos e Engenharia Rural do Centro de Ciências Agrárias da Universidade Federal da Paraíba, Areia, Paraíba, Brasil. O município está inserido na Microrregião do Brejo Paraibano, situado nas coordenadas de latitude $6^{\circ} 58^{\prime}$ $12^{\prime}$ ' $\mathrm{S}$, longitude $35^{\circ} 42^{\prime} 15^{\prime}$ ' $\mathrm{W}$ e altitude de 619 m. De acordo com a sistema de classificação de Köppen (ALVARES et al., 2013), o clima é do tipo 'As' (quente e úmido), com valores médios de umidade relativa e temperatura do ar, respectivamente, de $25^{\circ} \mathrm{C}$ e $75 \%$ nos meses mais quentes (outubro, novembro e dezembro) e de $21,6{ }^{\circ} \mathrm{C}$ e $87 \%$ nos meses mais frios (junho, julho e agosto).

Amostras de um solo salino-sódico, classificado como Neossolo Fúlvico (EMBRAPA, 2013), foram coletadas na profundidade de 0-0,2 m em toda a área do setor 7 do Perímetro de Irrigação de São Gonçalo, Sousa, PB. Em seguida foram destorroadas, homogeneizado e passado em peneira com malha de $2 \mathrm{~mm}$ e submetidas caracterização física de densidade, textura e química quanto à fertilidade utilizando o extrator acetato de amônio pH 7 (EMBRAPA, 2011) e quanto à salinidade do extrato de saturação (RICHARDS, 1954), conforme os resultados na Tabela 1.

Tabela 1. Caracterização dos atributos químicos quanto à fertilidade e salinidade e físicos do solo antes da aplicação dos tratamentos.

\begin{tabular}{lclllc}
\hline \multicolumn{1}{c}{ Fertilidade } & \multicolumn{3}{c}{ Salinidade } & \multicolumn{2}{c}{ Atributos físicos } \\
\hline $\mathrm{pH} \mathrm{em} \mathrm{H} \mathrm{H}_{2} \mathrm{O}(1: 2,5)$ & 10,40 & $\mathrm{pH}$ & 10,65 & Dens. do solo $\left(\mathrm{g} \mathrm{cm}^{-3}\right)$ & 1,69 \\
$\mathrm{P}\left(\mathrm{mg} \mathrm{dm}^{-3}\right)$ & 10,86 & $\mathrm{Ca}^{2+}\left(\mathrm{mmol}_{\mathrm{c}} \mathrm{L}^{-1}\right)$ & 1,50 & Dens. de partícula $\left(\mathrm{g} \mathrm{cm}^{-3}\right)$ & 2,82 \\
$\mathrm{~K}\left(\mathrm{cmol}_{\mathrm{c}} \mathrm{dm}^{-3}\right)$ & 0,12 & $\mathrm{Mg}^{2+}\left(\mathrm{mmol}_{\mathrm{c}} \mathrm{L}^{-1}\right)$ & 0,50 & Porosidade total $\left(\mathrm{m}^{3} \mathrm{~m}^{-3}\right)$ & 0,40 \\
$\mathrm{Ca}^{2+}\left(\mathrm{cmol}_{\mathrm{c}} \mathrm{dm}^{-3}\right)$ & 0,29 & $\mathrm{~K}^{+}\left(\mathrm{mmol}_{\mathrm{c}} \mathrm{L}^{-1}\right)$ & 0,15 & Areia $\left(\mathrm{g} \mathrm{kg}^{-1}\right)$ & 625,00 \\
$\mathrm{Mg}^{2+}\left(\mathrm{cmol}_{\mathrm{c}} \mathrm{dm}^{-3}\right)$ & 0,10 & $\mathrm{Na}^{+}\left(\mathrm{mmol}_{\mathrm{c}} \mathrm{L}^{-1}\right)$ & 177,70 & Silte $\left(\mathrm{g} \mathrm{kg}^{-1}\right)$ & 235,00 \\
\hline
\end{tabular}




\begin{tabular}{|c|c|c|c|c|c|}
\hline$\overline{\mathrm{Na}^{+}\left(\mathrm{cmol}_{\mathrm{c}} \mathrm{dm}^{-3}\right)}$ & 20,82 & $\mathrm{Cl}^{-}\left(\mathrm{mmol}_{\mathrm{c}} \mathrm{L}^{-1}\right)$ & 86,50 & Argila $\left(\mathrm{g} \mathrm{kg}^{-1}\right)$ & 140,00 \\
\hline $\mathrm{SB}\left(\mathrm{cmol}_{\mathrm{c}} \mathrm{dm}^{-3}\right)$ & 21,33 & $\mathrm{CO}_{3}^{2-}\left(\mathrm{mmol}_{\mathrm{c}} \mathrm{L}^{-1}\right)$ & 83,00 & Ada $\left(\mathrm{g} \mathrm{kg}^{-1}\right)$ & 90,00 \\
\hline $\mathrm{Al}^{3+}\left(\mathrm{cmol}_{\mathrm{c}} \mathrm{dm}^{-3}\right)$ & 0,00 & $\mathrm{HCO}_{3}{ }^{-}\left(\mathrm{mmol}_{\mathrm{c}} \mathrm{L}^{-1}\right)$ & 59,00 & Grau de floculação (\%) & 35,70 \\
\hline $\mathrm{H}^{+}+\mathrm{Al}^{3+}\left(\mathrm{cmol}_{\mathrm{c}} \mathrm{dm}^{-3}\right)$ & 0,00 & $\mathrm{SO}_{4}{ }^{2-}\left(\mathrm{mmol}_{\mathrm{c}} \mathrm{L}^{-1}\right)$ & 42,86 & Ucc $\left(\mathrm{g} \mathrm{kg}^{-1}\right)$ & 115,00 \\
\hline CTC $\left(\mathrm{cmol}_{\mathrm{c}} \mathrm{dm}^{-3}\right)$ & 21,33 & CEes $\left(\mathrm{dS} \mathrm{m}^{-1}\right)$ & 17,70 & Upmp ( $\left.\mathrm{g} \mathrm{kg}^{-1}\right)$ & 48,00 \\
\hline PST (\%) & 97,61 & RAS $\left(\mathrm{mmol} \mathrm{L}^{-1}\right)^{0,5}$ & 177,70 & Água disponível $\left(\mathrm{g} \mathrm{kg}^{-1}\right)$ & 67,00 \\
\hline $\mathrm{V}(\%)$ & 100,0 & PST (\%) & 99,18 & Classe textural: & FA \\
\hline Classificação: & Eut. & Classificação & SSS & --- & --- \\
\hline
\end{tabular}

$\mathrm{pH}=$ Potencial hidrogeniônico; $\mathrm{SB}=$ Soma de bases trocáveis $\left(\mathrm{Ca}^{2+}+\mathrm{Mg}^{2+}+\mathrm{K}^{+}\right) ; \mathrm{PST}=$ Percentagem de sódio trocável [(Na $\left./ \mathrm{CTC}\right]$ $\times 100]$; CTC $=$ Capacidade de troca de cátions $\left[\mathrm{SB}+\left(\mathrm{H}^{+}+\mathrm{Al}^{3+}\right)\right]$ acetato de amônio-neutro $(\mathrm{pH}-7,0) ; \mathrm{V}=$ Percentagem de saturação por bases trocáveis $[\mathrm{V}=(\mathrm{SB} / \mathrm{CTC}) \times 100]$; CEes = Condutividade elétrica do extrato de saturação ; SSS = Solo salino-sódico.

O material de solo do experimento foi passado em peneira com malha de $4 \mathrm{~mm}$ e acondicionado $4,21 \mathrm{~kg}$ em vasos plásticos com capacidade $5 \mathrm{~kg}$ com drenos na parte final inferior para a coleta do lixiviado. Os tratamentos foram distribuídos em blocos casualizados com três repetições e duas unidades por parcela usando o fatorial 5 $\times 3$, referente aos volumes de vinhaça aplicados ao solo nas proporções de $20,40,60,80$ e $100 \%$ do volume de água referente a umidade de capacidade de campo do solo em massa de $485 \mathrm{~mL}$ equivalentes a 97, 194, 291, 388 e $485 \mathrm{~mL}$ de vinhaça por vaso em três períodos de incubação de 10, 30 e 50 dias. O nível de umidade de capacidade de campo foi obtido com água até atingir o volume da dose de 100\% de vinhaça (485 $\mathrm{mL}$ ).

A vinhaça utilizada nos tratamentos foi proveniente do Engenho Quati, localizado no município de Pilões, Paraíba. Antes da aplicação dos tratamentos, alíquotas da vinhaça foram coletadas e analisadas como água de irrigação (Tabela 2), quanto aos atributos químicos, conforme Richards (1954) e na matéria seca conforme EMBRAPA (2011).

Tabela 2. Caracterização química da vinhaça utilizada no experimento.

\begin{tabular}{|c|c|c|c|c|c|c|}
\hline & \multicolumn{6}{|c|}{ Características químicas } \\
\hline $\mathrm{pH}$ & $\mathrm{CE}$ & MS & $\mathrm{MO}$ & $\mathrm{C}$ & $\mathrm{N}$ & $\mathrm{C}: \mathrm{N}$ \\
\hline \multirow{5}{*}{3,37} & $\mathrm{dS} \mathrm{m}^{-1}$ & \multicolumn{4}{|c|}{--------------------------g L L'------------------------- } & \multirow{2}{*}{ 13:1 } \\
\hline & 7,37 & 32,72 & 4,13 & 2,40 & 0,18 & \\
\hline & $\mathrm{P}$ & $\mathrm{K}^{+}$ & $\mathrm{Ca}^{2+}$ & $\mathrm{Mg}^{2+}$ & $\mathrm{Na}^{+}$ & \\
\hline & ------- & $\begin{array}{l}------1 \\
--1\end{array}$ & $L^{-1}---$ & $\begin{array}{l}------ \\
---1\end{array}$ & $\begin{array}{l}----- \\
--1\end{array}$ & \\
\hline & 0,06 & 1,50 & 0,30 & 0,20 & 152,44 & \\
\hline
\end{tabular}

$\mathrm{pH}=$ Potencial de hidrogeniônico; $\mathrm{CE}=$ Condutividade elétrica; $\mathrm{MS}=$ Massa seca; $\mathrm{MO}=$ Matéria orgânica; C = Carbono; $\mathrm{N}=$ Nitrogênio, C:N = Relação carbono/nitrogênio; P = Fósforo; $\mathrm{K}^{+}=$Potássio; $\mathrm{Ca}^{2+}=$ Cálcio; $\mathrm{Mg}^{2+}=$ Magnésio; $\mathrm{Na}^{+}=$Sódio.

Após cada período de incubação, o solo de cada tratamento foi submetido a uma lavagem com volume de água não salina [CEa $=0,4 \mathrm{dS} \mathrm{m}^{-1}$; RAS $\left.\left.=0,88(\mathrm{mmol} \mathrm{L})^{-1}\right)^{1 / 2}\right]$ correspondente a 1,5 vezes a umidade de saturação expressa pela porosidade total (Tabela 1) e ao cessar a drenagem foram determinados no volume lixiviado o $\mathrm{pH}$, $\mathrm{CE}$ e os teores de cátions $\left(\mathrm{K}^{+}, \mathrm{Ca}^{2+}, \mathrm{Mg}^{2+}, \mathrm{Na}^{+}\right)$ conforme Richards (1954), Brito et al. (2007) e Souto et al. (2015).

Os dados foram submetidos à análise de variância pelo teste $\mathrm{F}$ a $5 \%$ de probabilidade.
As médias referentes aos períodos de incubação foram comparadas pelo teste "Tukey" $(\mathrm{p}<0,05)$ e as relativas às doses de vinhaça regressão usando o software estatístico SISVAR 5.6 (FERREIRA, 2014).

\section{RESULTADOS E DISCUSSÃO}

A interação entre as doses de vinhaça e os períodos de incubação exerceu efeitos significativos na salinidade expressa pela condutividade elétrica dos lixiviados, isto é, 
pela composição química referente aos cátions solúveis (Tabela 3). O comportamento estatístico dos dados está em acordo com o registrado por outros autores como Brito et al. (2007) ao relatarem que a interação tipo de solo $\times$ dose de vinhaça $\times$ tempo de incubação interferiu significativamente na concentração de cátions presentes na solução drenada e
Santos et al. (2005) após avaliarem o lixiviado de um solo sódico tratado com gesso agrícola, composto de lixo urbano e vinhaça. Estão em consonância também com Yazdanpanah e Mahmoodabadi (2013) ao estudarem os efeitos da lâmina de lavagem associada à adição de matéria orgânica e gesso agrícola num solo salino-sódico.

Tabela 3. Resumo da análise de variância, pelo quadrado médio, relativos às variáveis químicas do lixiviado em função das doses de vinhaça e dos períodos de incubação em solo salino-sódico.

\begin{tabular}{|c|c|c|c|c|c|c|c|}
\hline \multirow{2}{*}{$\mathrm{FV}$} & \multirow{2}{*}{ GL } & \multicolumn{6}{|c|}{ Quadrado Médio } \\
\hline & & $\mathrm{pH}$ & $\mathrm{CE}$ & $\mathrm{K}^{+}$ & $\mathrm{Ca}^{2+}$ & $\mathrm{Mg}^{2+}$ & $\mathrm{Na}^{+}$ \\
\hline Blocos & 2 & $0,008^{\text {ns }}$ & $0,77^{\mathrm{ns}}$ & $0,0001^{\mathrm{ns}}$ & $0,002^{\mathrm{ns}}$ & $0,006^{\mathrm{ns}}$ & $34,69^{\text {ns }}$ \\
\hline Doses (D) & 5 & $0,21 * *$ & $25,77 * *$ & $0,0001^{\mathrm{ns}}$ & $2,45^{* *}$ & $0,19 * *$ & $1.702,99 * *$ \\
\hline Lin. & 1 & $0,79 * *$ & $68,46^{* *}$ & $0,000004^{\mathrm{ns}}$ & $9,35^{* *}$ & $0,72 * *$ & $3.864,84 * *$ \\
\hline Quad. & 1 & $0,03^{\mathrm{ns}}$ & $0,88^{\text {ns }}$ & $0,00019^{\mathrm{ns}}$ & $0,05^{\mathrm{ns}}$ & $0,02 * *$ & $719,11^{* *}$ \\
\hline Incub. (I) & 2 & $1,16^{* *}$ & $10,25 * *$ & $0,0018^{* *}$ & $5,07 * *$ & $0,34^{* *}$ & $1.908,15^{* *}$ \\
\hline $\mathrm{D} \times \mathrm{I}$ & 8 & $0,10^{* *}$ & $8,28 * *$ & $0,0016^{* *}$ & $1,34^{* *}$ & $0,15^{* *}$ & $781,78 * *$ \\
\hline Lin. ( $\left.\mathrm{I}_{1}\right)$ & 1 & $1,32 * *$ & $29,89 * *$ & $0,0012^{* *}$ & $18,44^{* *}$ & $1,53^{* *}$ & $4.745,84^{* *}$ \\
\hline Quad. (I $\left.\mathrm{I}_{1}\right)$ & 1 & $0,09^{\mathrm{ns}}$ & $1,58^{\mathrm{ns}}$ & $0,0020^{* *}$ & $0,44^{* *}$ & $0,03 * *$ & $473,53 * *$ \\
\hline Lin. $\left(\mathrm{I}_{2}\right)$ & 1 & $0,13^{* *}$ & $17,12^{* *}$ & $0,0001^{\mathrm{ns}}$ & $0,45^{* *}$ & $0,05^{* *}$ & $66,45^{\mathrm{ns}}$ \\
\hline Quad. (I $\left.\mathrm{I}_{2}\right)$ & 1 & $0,004^{\mathrm{ns}}$ & $3,41^{*}$ & $0,0002^{\text {ns }}$ & $0,20 * *$ & $0,01 *$ & $200,10^{*}$ \\
\hline Lin. $\left(\mathrm{I}_{3}\right)$ & 1 & $0,0006^{\mathrm{ns}}$ & $22,33^{* *}$ & $0,0007^{* *}$ & $0,11^{* *}$ & $0,00003^{\mathrm{ns}}$ & $938,56^{* *}$ \\
\hline Quad. (I3) & 1 & $0,001^{\mathrm{ns}}$ & $22,39 * *$ & $0,00003^{\mathrm{ns}}$ & $0,02^{\text {ns }}$ & $0,11^{* *}$ & $1.507,95^{* *}$ \\
\hline Resíduo & 28 & 0,02 & 0,77 & 0,00007 & 0,014 & 0,002 & 47,52 \\
\hline Total & 44 & & & & & & \\
\hline Média & & 9,20 & 13,31 & 0,05 & 1,32 & 0,62 & 67,47 \\
\hline dms (I) & & 0,135 & 0,796 & 0,0077 & 0,108 & 0,049 & 6,230 \\
\hline $\mathrm{CV}(\%)$ & & 1,63 & 6,62 & 16,50 & 9,10 & 8,80 & 10,22 \\
\hline
\end{tabular}

ns = não significativo; * = significativo à $5 \%$ de probabilidade; $* *=$ significativo à $1 \%$ de probabilidade; Lin = linear; Quad = quadrático; $\mathrm{I}_{1}$ = período de incubação de 10 dias; $\mathrm{I}_{2}=$ período de incubação de 30 dias; $\mathrm{I}_{3}$ = período de incubação de 30 dias.

Apesar do $\mathrm{pH}$ do lixiviado, exceto aos 50 dias, diminuir com o aumento das doses de vinhaça, os valores aumentaram com o período de incubação do insumo orgânico na seguinte ordem: $50>30>10$ dias (Figura 1A). A superioridade ao longo do tempo expressa melhoria física induzida pela matéria orgânica ao solo para a dinâmica da água (MELLEK et al., 2010) e melhoria química pelo deslocamento de sais como carbonatos de cálcio e de sódio, além de outros, que elevam o pH do lixiviado e, com efeito, diminui a alcalinidade no solo (MIRANDA et al., 2011; SOUSA et al., 2014). No solo incubado com vinhaça durante 10 e 30 dias o $\mathrm{pH}$ decresceu linearmente aos níveis de 0,0105 e 0,0434 por aumento unitário percentual de vinhaça incubada, promovendo declínios de 9,3 para 8,5 e de 9,4 para 9,1 correspondentes a diminuições de 9,6 e 3,2\%, entre os tratamentos incubados com a maior e menor dose de vinhaça, nos respectivos períodos. Nas mesmas doses de vinhaça incubadas por 50 dias, os dados do $\mathrm{pH}$ não se ajustaram a nenhum modelo de regressão com maior valor médio de 9,47.

A redução do $\mathrm{pH}$ no lixiviado do solo salino-sódico, em função das doses de vinhaça é atribuída a presença e liberação de ácidos orgânicos e $\mathrm{CO}_{2}$, principalmente nas maiores concentrações do composto orgânico durante a decomposição anaeróbica, o que contribui para a acidificação da solução e consequentemente redução do $\mathrm{pH}$ 
(NICOCHELLI et al., 2012; PRAPAGAR et al., 2012). Essa situação está corroborando com a composição química devido a vinhaça apresentar caráter ácido com $\mathrm{pH}$ de 3,37, conforme verificado na Tabela 2. Quanto ao periodo de incubação, os resultados assemelham-se aos obtidos por Santos et al. (2005) após constatarem maiores valores no $\mathrm{pH}$ do lixiviado de um solo-sódico tratado com gesso, lixo urbano e vinhaça, aos 52 dias de incubação. No tocante aos efeitos da matéria orgânica, os valores estão coerentes com Negim e Mustafa (2016) ao registrarem diminuição do $\mathrm{pH}$ da solução drenada de um solo salino-sódico, tratado com torta de filtro da cana-de-açúcar e ácido fosfórico incubado por período de 30 dias, de 8,34 para 7,41 e declínio de 11,2\%.

O aumento das doses de vinhaça, exceto no maior período de incubação (50 dias) que exerceu declínio com o aumento das doses até 59,5\%, promoveu aumento da condutividade elétrica da solução lixiviada do solo salino-sódico, aos 10 e 30 dias de incubação (Figura 1B). Os aumentos foram, respectivamente, de 11,97 para 16,43 $\mathrm{dS} \mathrm{m}^{-1} \mathrm{e}$ de 10,80 para $12,60 \mathrm{dS} \mathrm{m} \mathrm{m}^{-1}$, aos 10 e 30 dias de incubação da vinhaça, com expressivos aumentos na lixiviação dos sais do solo de 37,3 e $16,7 \%$ entre os tratamentos com 20 e $100 \%$ da dose de vinhaça fornecida.
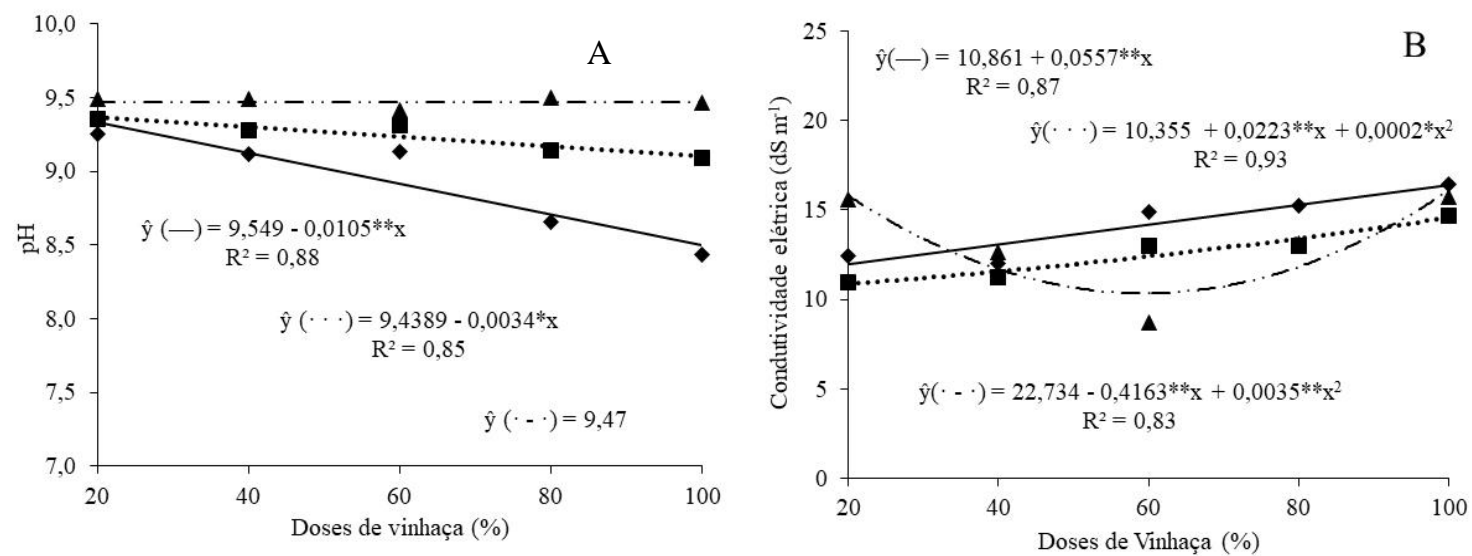

Figura 1. Valores de pH (A) e condutividade elétrica (B) do lixiviado de um solo salino-sódico incubado 10 dias (一), 30 dias $(\cdots)$ e 50 dias $(\cdot-\cdot)$, em função de doses de vinhaça.

Os aumentos na condutividade elétrica da solução lixiviada com o aumento das doses de insumo orgânico aplicado está em acordo com o aumento do $\mathrm{pH}$ do solo nas mesmas condições e é resultado da melhoria física do solo pela estrutura e espaço poroso, dinâmica de água, substituição do sódio trocável pelo cálcio e lixiviação dos sais do solo e, consequentemente, acúmulo no lixiviado (TAZEH et al., 2013). Como, inclusive, concluíram também Vasconcelos et al. (2016) ao avaliarem a condutividade elétrica da água drenada de um Neossolo Flúvico com problema de salinidade e sodicidade cultivado com feijão caupi (Vigna unguiculata) em resposta às doses de gesso agrícola e lâminas de irrigação.

O aumento das doses de vinhaça promoveu a lixiviação de potássio, ao longo do período de incubação no solo salino-sódico expressando diminuição do cátion na solução drenada, na ordem: dez dias maior que cinquenta e este maior que trintas dias (Figura 2A). No solo incubado com vinhaça durante 10 dias, o aumento das doses de vinhaça de $58 \%$ elevou a lixiviação de $\mathrm{K}^{+}$até o maior valor de 0,0734 $\mathrm{mmol}_{\mathrm{c}} \mathrm{L}^{-1}$. Nas mesmas condições, no solo incubado por 30 dias os teores de potássio na solução drenada não se adequaram a nenhum tipo de regressão, sendo representado pelo valor médio de 0,0407 mmol $_{c} \mathrm{~L}^{-1}$ de $\mathrm{K}^{+}$. No solo incubado com vinhaça por 50 dias, os teores no lixiviado foram reduzidos linearmente de 0,0854 para 0,0454 mmol $_{\mathrm{c}} \mathrm{L}^{-1}$ de $\mathrm{K}^{+}$entre as doses de 20 e $100 \%$, com lixiviação de 46,7\% entre os tratamentos com 20 e $100 \%$ da dose de vinhaça incubada ao solo. 
O comportamento dos dados é semelhante ao apresentado por Brito et al. (2005) após aplicarem vinhaça em três solos (Nitossolo, Argissolo e Espodossolo) e constatarem diminuição do potássio na solução drenada. A redução de potássio no lixiviado que indica lixiviação do elemento do solo depende de fatores como a dose e a composição de bases trocáveis do insumo, do tempo de incubação ao solo, características de adsorção de íons, maior ou menor migração de bases trocáveis e pela facilidade de contato entre o soluto presente na vinhaça e a superfície de adsorção do solo (BRITO et al., 2005; NICOCHELLI et al., 2012; ORTEGÓN et al., 2016).

A concentração de $\mathrm{Ca}^{2+}$ no lixiviado foi elevada com o aumento das doses de vinhaça aplicada ao solo salino-sódico (Tabela 1) sendo a maior proporção lixiviada dos tratamentos com o menor tempo de incubação de 10 dias (Figura 2B). A redução entre as doses de 20 e $100 \%$ indicaram que o maior período de incubação resultou em menor lixiviação de cálcio. Esses resultados estão em acordo com os apresentados por Santos et al. (2005) em solo sódico tratado com gesso agrícola, composto de lixo urbano e cultivado com algodão (Gossypium hirsutum L.), ao descreverem que o aumento do período de incubação do solo com vinhaça de 15 para 60 dias, reduziu a perda de cálcio na solução drenada.

Os valores no lixiviado para a vinhaça incubada durante 30 dias foram elevados linearmente em $0,0034 \mathrm{mmol}_{\mathrm{C}} \mathrm{L}^{-1}$ por unidade da dose de vinhaça, com maior valor de 0,9624 mmol $_{\mathrm{c}} \mathrm{L}^{-1}$ na dose de $100 \%$, ao comparar esse resultado com o valor 0,6904 mmolc $_{\mathrm{C}} \mathrm{L}^{-1}$ a menor dose (20\% de vinhaça), a elevação foi de 39,4 \% e evidência a importância do aumento insumo orgânico para correção da sodicidade dos solos. Comportamento semelhante foi verificado nos tratamentos do solo com vinhaça incubada por 50 dias em que os teores de $\mathrm{Ca}^{2+}$ aumentaram de 0,8426 para 1,2426 mmol $_{\mathrm{c}} \mathrm{L}^{-1}$, com incremento de 38,31 $\%$ entre a maior e a menor dose do insumo orgânico aplicado.
A aplicação de matéria orgânica, como é o caso da vinhaça, pode melhorar as propriedades químicas de um solo com problema de salinidade e sodicidade, principalmente devido à substituição de $\mathrm{Na}^{+}$ do solo pelo $\mathrm{Ca}^{2+}$ do corretivo químico ou insumo orgânico e, consequentemente, remoção do sódio trocável durante a lavagem do solo (Santos et al., 2005). No entanto, vale ressaltar que deve ser bem definida a dose de vinhaça aplicada ao solo salino-sódico, pois a remoção dos sais vai depender da composição química do insumo, uma vez que concentrações elevadas podem conter maiores quantidades de elementos do que a capacidade de troca de cátion que o solo possui, contribuindo para elevadas perdas, por exemplo, de $\mathrm{Ca}^{2+}$ durante o processo de lixiviação (JALALI; RANJBAR, 2009; YAZDANPANAH et al., 2011).

Comportamento semelhante ao cálcio foi observado para o magnésio, onde o aumento das doses de vinhaça aplicadas ao solo, também contribuiu para a maior lixiviação de $\mathrm{Mg}^{2+}$ com destaque para o período de incubação de 10 dias (Figura 2C), em que os teores foram aumentados de 0,3853 para $1,4593 \mathrm{mmol}_{\mathrm{c}} \mathrm{L}^{-1}$ entre os tratamentos com vinhaça aos níveis de 20 para $100 \%$. Nos tratamentos do solo com vinhaça incubada por 30 e 50 dias, os teores de $\mathrm{Mg}^{2+}$ foram elevados até as doses máximas estimadas de 83 e 44,5\%, com os maiores valores de 0,5453 e 0,6122 mmolc $_{c} \mathrm{~L}^{-1}$, respectivamente. Tendência semelhante foi verificada por Negim e Mustafa (2016) ao registrarem o aumento nos teores de magnésio no lixiviado do solo e atribuíram a liberação do elemento à medida que se elevou a decomposição e mineralização do insumo orgânico aplicado.

A elevação das doses de vinhaça aplicada ao solo proporcionou a maior lixiviação de sódio com o aumento do período de incubação exibindo a ordem de: 10 dias> 30 dias $>50$ dias, como verificado na Figura 2D. O aumento das doses de vinhaça, durante os períodos de incubação de 10 e 30 dias, até 94,5 e 58 \% promoveu a maior lixiviação de sódio do solo com os maiores valores, respectivamente de 98,8586 e 57,2722 $\mathrm{mmol}_{\mathrm{c}}$ 
$\mathrm{L}^{-1}$. Durante o período de incubação de 50 dias, o aumento das doses de vinhaça de 20 até $68,5 \%$ promoveu redução do sódio no lixiviado maiores teores de sódio lixiviado foram aos tratamentos com a aplicação $100 \%$ da dose de vinhaça, onde atingiu o valor máximo de 61,4247 mmolc $\mathrm{L}^{-1}$. Possivelmente, tenha ocorrido a substituição de $\mathrm{Ca}^{2+}$ por $\mathrm{Na}^{+}$no complexo de troca, tendo em vista que ocorreu redução nos teores de potássio (Figura 2A e 2B) em detrimento da elevação nos teores de sódio do lixiviado (Figura 2D) com aumento da concentração de vinhaça aplicada ao solo.

Pelos resultados, o tempo de incubação de 10 dias foi o mais eficiente na remoção dos íons de sódio trocável do solo tratado com vinhaça. Os valores máximo estimando do elemento na suspensão de cada período de incubação, verifica-se que a remoção de sódio do solo salino-sódico pelo período de 10 dias foi superior em $72,61 \%$ ao período de 30 dias e $60,94 \%$ ao período de 50 dias. Resultados semelhantes são descritos por Brito et al. (2007) ao constatarem que a aplicação de vinhaça e incubação do solo por período de 30 dias promove maior remoção do íon em relação ao período de 60 dias. Ao avaliarem a aplicação de condicionadores orgânicos, Miranda et al. (2011) obtiveram maiores teores de $\mathrm{Na}^{+}$na solução lixiviada nos solos incubados por sete dias em comparação aqueles incubados por 70 dias.
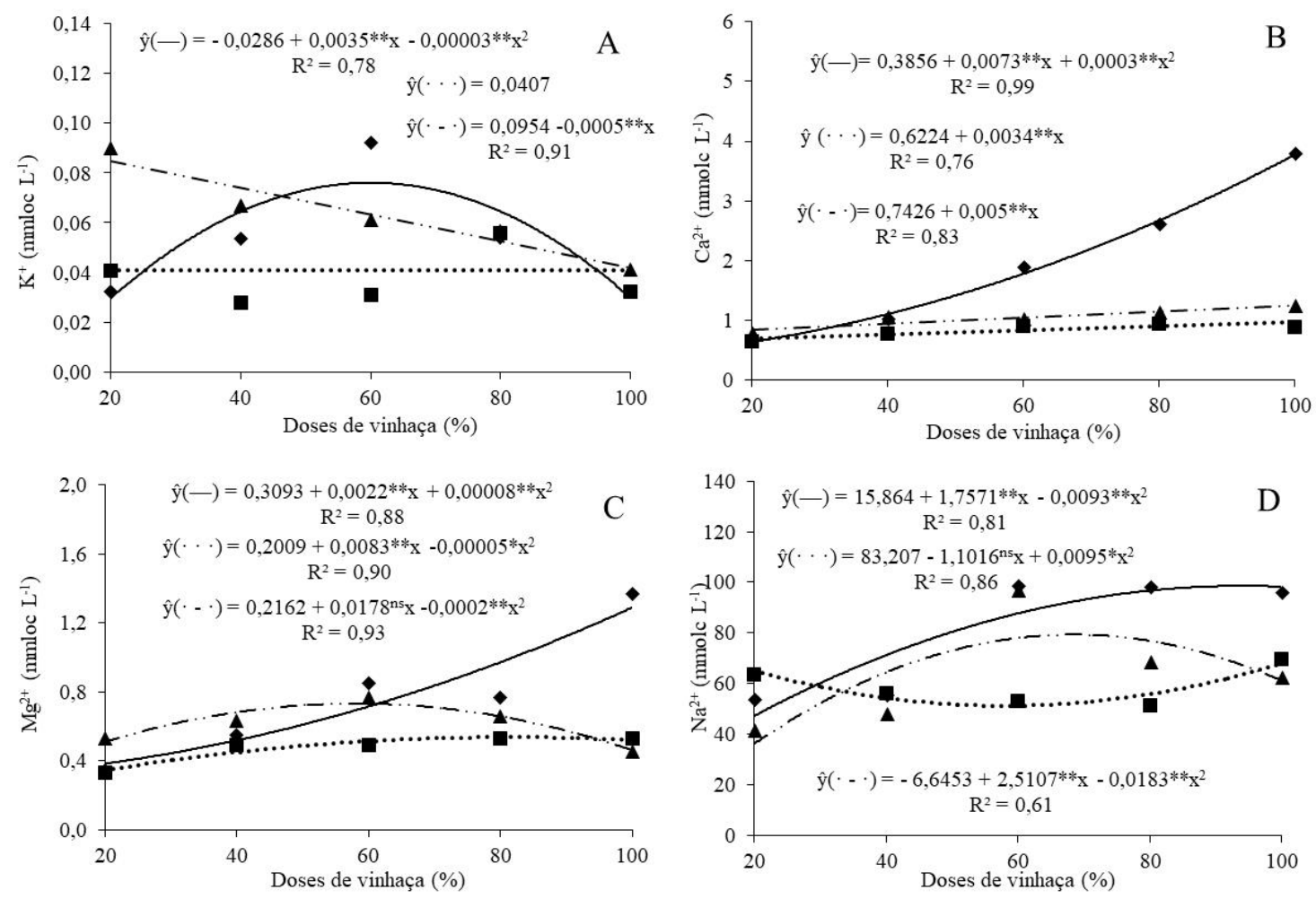

Figura 2. Concentração de potássio (A), cálcio (B), magnésio (C) e sódio (D) no lixiviado de um solo salinosódico incubado com vinhaça por $10(-), 30(\cdots)$ e 50 dias $(\cdot \cdot \cdot)$.

\section{CONCLUSÕES}

A redução do $\mathrm{pH}$, aumento da condutividade elétrica e dos teores catiônicos de $\mathrm{Na}^{+}, \mathrm{Ca}^{2+}, \mathrm{Mg}^{2+}$ e $\mathrm{K}^{+}$, em função das doses e diminuição com o aumento do período de incubação, indica ação positiva da vinhaça na redução da salinidade, da sodicidade e alcalinidade do solo degradado por sódio trocável;

O periodo de incubação da vinhaça por dez dias promoveu a maior redução de todas as variáveis estudadas.

A dose de $100 \%$ do insumo orgânico resultou em maior redução de $\mathrm{pH}$, condutividade elétrica, cálcio e magnésio e as 
de 58 e 95\% de potássio e sódio, respectivamente.

\section{REFERÊNCIAS BIBLIOGRÁFICAS}

ABDEL-FATTAH, M. K. Role of gypsum and compost in reclaiming salinesodic soils. Journal of Agriculture and Veterinary Science, Al Qasim, v. 1, n. 3, p. 30-38, 2012.

ALVARES, C. A.; STAPE, J. L.; SENTELHAS, P. C.; DE MORAES GONÇALVES, J. L.; SPAROVEK, G. Köppen's climate classification map for Brazil. Meteorologische Zeitschrift, Berlin, v. 22, n. 6, p. 711-728, 2013.

BRITO, F. L.; ROLIM, M. M.; PEDROSA, E. M. R. Concentração de cátions presentes no lixiviado de solos tratados com vinhaça. Revista Engenharia Agrícola, Jaboticabal, v. 27, n. 3, p. 773-781, 2007.

BRITO, F. L.; ROLIM, M. M.; PEDROSA, E. M. R. Teores de potássio e sódio no lixiviado e em solos após a aplicação de vinhaça. Revista Brasileira de Engenharia Agrícola e Ambiental, Campina Grande, v. 9, n. supl., p. 52-56, 2005.

BUENO, P. C.; RUBÍ, J. A. M.; GIMÉNEZ, R. G.; BALLESTA, R. J. Impacts caused by the addition of wine vinasse on some chemical and mineralogical properties of a Luvisol and a Vertisol in La Mancha (Central Spain). Journal of Soils and Sediments, Berlin, v. 9, n. 2. p. 121-128, 2009.

CAVALCANTE, L. F.; SANTOS, R. V.; HERNANDEZ, F. F. F.; GHEYI, H. R.; DIAS, T. J.; NUNES, J. C.; LIMA, G. S. Recuperação de solos afetados por sais. In: GHEYI, H. R.; DIAS, N. S.; LACERDA, C. F.; GOMES FILHO, E. Manejo da salinidade na agricultura: Estudos básicos e aplicados. 2. ed. Fortaleza: INCTSal, 2016. cap. 28, p. 461-479.
EMBRAPA - Empresa Brasileira De Pesquisa Agropecuária. Sistema Brasileiro de Classificação de Solos. 3. ed. Rio de Janeiro: Embrapa Solos, 2013. 353 p.

EMBRAPA - Empresa Brasileira De Pesquisa Agropecuária. Manual de métodos de análise do solo. 2. ed. Rio de Janeiro: Embrapa Solos, 2011, 230 p.

FERREIRA, D. F. Sisvar: a Guide for its Bootstrap procedures in multiple comparisons. Ciência e Agrotecnologia, Lavras, v. 38, n. 2, p. 109-112, 2014.

GOMES, E. M.; GHEYI, H. R.; SILVA, E. F. F. Melhorias nas propriedades químicas de um solo salino-sódico e rendimento de arroz, sob diferentes tratamentos. Revista Brasileira de Engenharia Agrícola e Ambiental, Campina Grande, v. 4, n. 3, p. 355-361, 2000.

JALALI, M.; RANJBAR, F. Effects of sodic water on soil sodicity and nutrient leaching in poultry and sheep manure amended soils. Geoderma, Amsterdam, v. 153, n.1-2, p. 194204, 2009.

MIRANDA, M. A.; OLIVEIRA, E. E. M.; SANTOS, K. C. F.; FREIRE, M. B. G. S.; ALMEIDA, B. G. Condicionadores químicos e orgânicos na recuperação de solo salinosódico em casa de vegetação. Revista Brasileira de Engenharia Agrícola e Ambiental, Campina Grande, v. 15, n. 5, p. 484-490, 2011.

MELLEK, J. E.; DIECKOW, J.; SILVA, V. L.; FAVARETTO, N.; PAULETTI, V.; VEZZANI, F. M.; SOUZA, J. L. M. Dairy liquid manure and no-tillage: Physical and hydraulic properties and carbon stocks in a Cambisol of Southern Brazil. Soil \& Tillage Research, Amsterdam, v. 110, n.1, p. 69-76, 2010.

NEGIM, O.; MUSTAFA, A. Remediation of a highly calcareous saline sodic soil using some soil amendments. International 
Journal of Plant \& Soil Science, Hooghly, v. 12, n. 5, p. 1-13, 2016.

NICOCHELLI, L. M.; NASCENTES, R.; LIMA, E. B. N. R.; SOARES, F. S. C. Sorção de potássio em amostras de solo submetidas à aplicação de vinhaça. Revista Brasileira de Engenharia Agrícola e Ambiental, Campina Grande, v. 16, n. 7, p. 754-760, 2012.

ORTEGÓN, G. P.; ARBOLEDA, F. M.; CANDELA, L.; TAMOH, K.; VALDES ABELLAN, J. Vinasse application to sugar cane fields. Effect on the unsaturated zone and groundwater at Valle del Cauca (Colombia). Science of the Total Environment, Amsterdam, v. 539, n. 1, p. 410-419, 2016.

PARIHAR, P.; SINGH, S.; SINGH, R.; SINGH, V. P.; PRASAD, S. M. Effect of salinity stress on plants and its tolerance strategies: a review. Environmental Science and Pollution Research, Landsberg, v. 22, n. 6, p. 4056-4075, 2015.

PESSOA, L. G. M.; OLIVEIRA, E. E. M.; FREIRE, M. B. G. S.; FREIRE, F. J.; MIRANDA, M. A.; SANTOS, R. L. Composição química e salinidade do lixiviado em dois solos cultivados com cebola irrigada com água salina. Revista Brasileira de Ciências Agrárias, Recife, v. 5, n. 3, p. 406412, 2010.

PRAPAGAR, F.; INDRARATNE, S. P.; PREMANANDHARAJAH, P. Effect of soil amendments on reclamation of saline-sodic soil. Tropical Agricultural Research, Peradeniya, v. 23, n. 2, p. 168-176, 2012.

RIBEIRO, M. R.; RIBEIRO FILHO, M. R.; JACOMINE, P. K. T. Origem e classificação dos solos afetados por sais. In: GHEYI, H. R.; DIAS, N. S.; LACERDA, C. F.; GOMES FILHO, E. Manejo da salinidade na agricultura: Estudos básicos e aplicados. $2^{\circ}$ ed. Fortaleza: INCTSal, 2016. cap. 2, p. 9-15.
RICHARDS, L. A. Diagnosis and improvement of saline and alkaline soils. Washington: United States Salinity Laboratory Staff, 1954. 160 p. (Agriculture, $60)$.

SANTOS, M. F. G.; OLIVEIRA, F. A.; CAVALCANTE, L. F.; MEDEIROS, J. F.; SOUZA, C. C. Solo sódico tratado com gesso agrícola, composto de lixo urbano e vinhaça.

Revista Brasileira de Engenharia Agrícola e Ambiental, Campina Grande, v.9, n.3, p.307-313, 2005.

SOUSA, C. H. C.; LACERDA, C. F.; SILVA, F. L. B.; NEVES, A. L. R.; COSTA, R. N. T. GHEYI, H. R. Yield of cotton/cowpea and sunflower/cowpea crop rotation systems during the reclamation process of a salinesodic soil. Engenharia Agrícola, Jaboticabal, v. 34, n. 5, p. 867-876, 2014.

SOUTO, A. G. L.; CAVALCANTE, L. F.; GHEYI, R. H.; NUNES, J. C.; OLIVEIRA, F. I. F.; ORESCA, D. Photosynthetic pigments and biomass in noni irrigated with saline waters with and without leaching. Revista Brasileira de Engenharia Agrícola e Ambiental, Campina Grande, v. 19, n. 11, p. 1042-1048, 2015.

TAZEH, E. S.; PAZIRA, E.; NEYSHABOURI, M. H.; ABBASI, F.; ABYANEH, H. Z. Effects of two organic amendments on EC, SAR and soluble ions concentration in a saline-sodic soil. International Journal of Biosciences, Rajshahi, v. 3, n. 9, p. 55-68, 2013.

VASCONCELOS, R. R. A.; GRACIANO, E. S. A.; FONTENELE, A. J. P. B.; CORDEIRO NETO, A. T.; BARROS, M. F. C. Qualidade da água drenada e desenvolvimento do feijãocaupi em solos salino-sódicos após uso de gesso associado à lâmina de lixiviação. Revista Brasileira de Agricultura Irrigada, Fortaleza, v.10, n. 3, p. 640-650, 2016.

YAZDANPANAH, N.; MAHMOODABADI, M. Reclamation of calcareous saline-sodic 
soil using different amendments: Time changes of soluble cations in leachate. Arabian Journal of Geosciences, Riyadh, v. 6, n. 7, p. 2519-2528, 2013.

YAZDANPANAH, N.; PAZIRA, E.;
NESHAT, A.; NAGHAVI, H.; MOEZI, A. A.; MAHMOODABADI, M. Effect of some amendments on leachate properties of a calcareous saline-sodic soil. International Agrophysics, Lublin, v. 25, n.3, p. 307-310, 2011. 